\title{
Nonequilibrium wetting of finite samples
}

\author{
Thomas Kissinger, Andreas Kotowicz, Oliver Kurz, \\ Francesco Ginelli and Haye Hinrichsen \\ Fakultät für Physik und Astronomie, Universität Würzburg \\ D-97074 Würzburg, Germany
}

\begin{abstract}
As a canonical model for wetting far from thermal equilibrium we study a Kardar-Parisi-Zhang interface growing on top of a hard-core substrate. Depending on the average growth velocity the model exhibits a non-equilibrium wetting transition which is characterized by an additional surface critical exponent $\theta$. Simulating the single-step model in one spatial dimension we provide accurate numerical estimates for $\theta$ and investigate the distribution of contact points between the substrate and the interface as a function of time. Moreover, we study the influence of finite-size effects, in particular the time needed until a finite substrate is completely covered by the wetting layer for the first time.
\end{abstract}

\section{Introduction}

When a chemically inert surface is exposed to a gas phase particles may preferentially attach to the surface, forming a wetting layer of a different phase. By changing physical parameters such as pressure or temperature these systems may undergo a wetting transition at which the thickness of the layer diverges and becomes macroscopic. In many experimental situations it is reasonable to assume that the wetting layer is in thermal equilibrium with the substrate so that methods of equilibrium statistical physics can be applied $[1,2]$. More recently, however, wetting phenomena far from equilibrium attracted considerable attention [3-9]. These studies are motivated by the question whether wetting processes under non-equilibrium conditions, for example caused by a flux of particles or energy, may show physical properties that are qualitatively different from those of equilibrium wetting, especially at or in the vicinity of a wetting transition.

In order to describe a wetting process far from equilibrium one has to resort to a dynamical description of the microscopic processes. This can be done either by introducing explicit solid-on-solid growth models $[4,5]$ where the microscopical dynamics is described by appropriate rules for deposition and absorption of adatoms over the substrate, or by studying appropriate Langevin equations for non-equilibrium growth [8]. Concerning their universal properties all these studies were so far related to the Kardar- 


\begin{tabular}{|c||c|c|c|c|}
\hline & $\alpha$ & $\beta$ & $z$ & $\theta$ \\
\hline$\lambda<0$ & $1 / 2$ & $1 / 3$ & $3 / 2$ & $1.184(10)$ \\
$\lambda=0$ & $1 / 2$ & $1 / 4$ & 2 & $3 / 4$ \\
$\lambda>0$ & $1 / 2$ & $1 / 3$ & $3 / 2$ & $0.228(5)$ \\
\hline
\end{tabular}

Table 1. Critical exponents of wetting transitions in one spatial dimension

Parisi-Zhang equation [10] in one spatial dimension with an additional potential term [3]

$$
\frac{\partial h(\vec{x}, t)}{\partial t}=v_{0}+\sigma \nabla^{2} h(\vec{x}, t)-\frac{\partial V(h(\vec{x}, t))}{\partial h(\vec{x}, t)}+\lambda(\nabla h(\vec{x}, t))^{2}+\zeta(\vec{x}, t),
$$

where $h(\vec{x}, t)$ is the height of the interface defining the thickness of the growing layer, $v_{0}, \sigma$ and $\lambda$ are real parameters and $\zeta(\vec{x}, t)$ is a white Gaussian noise. Here $V(h)$ is a potential that takes the influence of the substrate into account. The nonequilibrium properties of this equation are caused by the non-linear term $\lambda(\nabla h)^{2}$ which can be shown to be a relevant perturbation in the renormalization group sense that violates detailed balance. Obviously this term breaks reflection symmetry $h \rightarrow-h$ so that in combination with a symmetry-breaking substrate the sign of $\lambda$ is expected to play a significant role. In the special case $\lambda=0$, however, it can be shown that the dynamics in a stationary state obeys detailed balance and is thus at thermal equilibrium. Hence by varying $\lambda$ we can study the crossover from equilibrium to nonequilibrium wetting.

Without a substrate, i.e., for $V=0$, a one-dimensional interface evolving according to the KPZ equation is known to roughen as $w(t) \sim t^{\beta}$, where $w(t)$ is the width (defined as the standard deviation of the heights) and $\beta$ is the so-called growth exponent. In a finite system of size $L$ this roughening process continues until the width saturates at $w_{\mathrm{s}} \sim L^{\alpha}$ when the intrinsic correlation length $\xi \sim t^{1 / z}$ reaches the system size. Here $z$ and $\alpha=z \beta$ are the dynamical exponent and the roughness exponent, respectively. In the case of KPZ growth in one spatial dimension, where a fluctuation-dissipation theorem holds, the exponents are given by simple rational values [11], see Table 11.

In order to study nonequilibrium wetting, one has to introduce a substrate by imposing a boundary in the microscopic dynamics or by choosing an appropriate form of the potential appearing in Eq. (11). The simplest way to introduce such a boundary is to impose the restriction $h(\vec{x}, t) \geq 0$, corresponding to an infinite-step potential of the form

$$
V(h)=\left\{\begin{array}{ll}
0 & \text { if } h \geq 0 \\
\infty & \text { if } h<0
\end{array} .\right.
$$

In order to avoid singularities in the continuous formulation, this infinite-step potential is usually replaced in Eq. (11) by an exponential function

$$
V(h)=\exp (-h)
$$




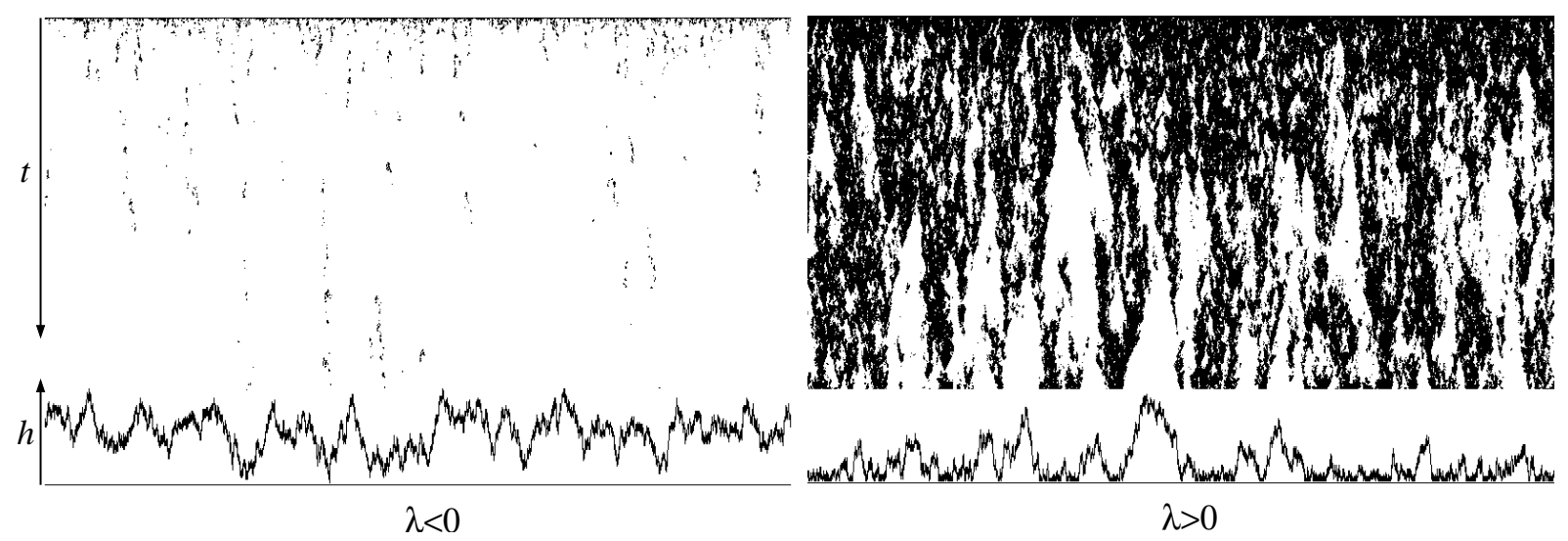

Figure 1. Upper row: Typical spatio-temporal pattern of contact points (pinned sites) between interface and substrate in the SSM (see text) at criticality, starting with a flat interface at $t=0$. Lower row: Final configuration of the interface at $t=5000$. Note that for $\lambda<0$ there are only few contact points, while for $\lambda>0$ the density of contact points is much higher.

that was found to display essentially the same physical properties.

Models defined by the addition of this simple boundary to a KPZ roughening surface are known to exhibit a continuous wetting transition. In an infinite system the critical point of this transition is determined by the asymptotic average velocity $v_{\infty}=\lim _{t \rightarrow \infty} v(t)$ of a free interface. For $v_{\infty}>0$ the interface moves away from the substrate so that it no longer influences the dynamics. For $v_{\infty}<0$, however, the interface is driven towards the substrate, approaching a steady state of finite roughness that is characterized by a certain height profile. At the transition point, where $v_{\infty}=0$, a scale-invariant behavior is observed. Obviously, the order parameter that characterizes this transition is the density of sites where the interface touches the substrate

$$
\rho_{s}(t)=\frac{1}{L} \sum_{i=1}^{L} \delta_{h_{i}(t), 0} .
$$

or - equivalently - the spatial average $\overline{\exp (-h(\vec{x}, t))}$ in the continuous formulation.

From a theoretical point of view the influence of a hard-core substrate without additional short-range forces can be interpreted as a boundary condition in the space spanned by $\vec{x}, t$, and $h$. As in other critical phenomena, this boundary condition does not change the underlying universality class and the associated bulk exponents, meaning that the process still belongs to the KPZ universality class. However, the substrate as an additional boundary condition adds a new feature, characterized by an order parameter $\rho_{s}(t)$ which is associated with additional surface exponent $\theta$. This order parameter was found decrease with increasing time as a power law [3]

$$
\rho_{s}(t) \sim t^{-\theta}
$$

The exponent $\theta$ is expected to take three different values depending on the sign of 
the parameter $\lambda$ of the nonlinear term. In fact, already a visual inspection of typical interfaces at criticality reveals significant differences depending on the sign of $\lambda$. As can be seen in Fig. 1 for $\lambda<0$ only few contact points remain, leading to a fast decay of $\rho_{s}(t)$, while for $\lambda>0$ the density of contact points decays only slowly.

Quite interestingly, it has recently been discovered, both through numerical simulations and analytical arguments $[12,13]$, that also the complete synchronization phase transition (CST) in most spatially extended chaotic dynamical systems belongs to the same universality class of a bounded KPZ equation with a negative nonlinear term $\lambda$. It can be shown that, when the local dynamic is smooth enough (more precisely: the linearization of the system around the synchronized state correctly describes the propagation of finite perturbations), the critical behavior of the CST is described by the so called multiplicative noise (MN) Langevin equation

$$
\frac{\partial}{\partial t} n(\vec{x}, t)=a n(\vec{x}, t)-n^{2}(\vec{x}, t)+D \nabla^{2} n(\vec{x}, t)+n(\vec{x}, t) \zeta(\vec{x}, t)
$$

where the order parameter $n(\vec{x}, t) \geq 0$ is the coarse grained density of unsynchronized regions. Here $a$ and $D>0$ are real parameters, and $\zeta(\vec{x}, t)$ is the same noise as in Eq. (11). By a Cole-Hopf transformation [3]

$$
h(\vec{x}, t)-\log n(\vec{x}, t)
$$

Eq. (6) turns exactly into the bounded KPZ equation (11) with $\lambda=-\sigma=-D$ and an exponential potential as in Eq. (3). This indicates that the MN equation is just a realization of the KPZ universality class and its non-trivial surface critical behavior. In particular the order parameter $n(\vec{x}, t)$ in Eq. (6) scales exactly in the same way as the density of contact points $\rho_{s}(t)$ and therefore its spatial average will decay as $t^{-\theta}$.

Eq. (6) has been studied numerically in a series of papers [15-17] and its transition is said to belong to the multiplicative noise I (MN1) class. Moreover, the related multiplicative Langevin equation which can be derived via an inverse Cole-Hopf transformation from the bounded KPZ equation (11) with $\lambda>0$ has been numerically analyzed in Ref. [18]. The associated transition is said to belong to the multiplicative noise II (MN2) class.

Presently, no exact analytical derivation of the surface exponent $\theta$ in the nonequilibrium case is known. While in Ref. [19] the surface exponent has been obtained for both the MN1 and MN2 classes with a mean-field like approximation suited for one spatial dimension, the presence of a strong-coupling fixed point and of essential divergences makes the exact values of the surface exponent not accessible by known renormalization group techniques. On the other hand, as we shall see, the equilibrium case $\lambda=0$, sometimes referred to as bounded Edward-Wilkinson, yields the simple rational result for the surface exponent $\theta_{E W}=3 / 4$. In light of this result, it would be desirable to know if also the nonequilibrium surface exponents have simple rational values, an indication that the surface critical behavior is a trivial consequence of the 
known KPZ bulk properties. In particular, in Ref. [20] the value $\theta=7 / 6$ has been conjectured for $\lambda<0$. Moreover, it is not yet fully clear how the contact points between interface and substrate are distributed and how finite-size effects influence the dynamics at the substrate.

As a first step toward a better comprehension of the bounded KPZ class, we present here a detailed analysis of a simple solid on solid model generally belonging to the KPZ universality class, the so-called single step model (SSM). Once provided with an additional boundary restriction without additional short-range forces, it shows a simple wetting transition belonging to the bounded KPZ universality class [21]. In particular, the simplicity of the model allows for an analytical knowledge of it's wetting transition critical point $v_{\infty}=0$. In Sect. 2 the efficiency of its algorithm allows us to improve current numerical estimates of the exponent $\theta$.

In Sect. 3 we investigate the distribution of intervals between the contact points, identifying significantly different scaling behaviors depending on the sign of $\lambda$. Finally, in Sect. 目 we study the scaling properties of finite systems, analyzing the time that is needed before the interface detaches globally for the first time. This kind of finite size scaling analysis is a common tool in numerical studies of nonequilibrium systems with an absorbing state, such as Directed Percolation (DP) [22], where the average absorbing time is known to scale according to the dynamical exponent, $\tau \sim L^{z}$. However, supported by numerical results and analytical arguments, we argue that in the case $\lambda \leq 0$ this detachment time does not grow as a power law with the system size. Conclusions are drawn in Sect. [5 while in Appendix A we analytically compute the surface exponent when detailed balance is imposed in the SSM, thus proving the equilibrium result $\theta_{E W}=3 / 4$.

\section{Measurement of the surface exponent in the single step model}

In what follows let us consider the so-called single step model (SSM) in one spatial dimension, which is probably the simplest and most compelling lattice model for KPZtype interface growth. In this model the growing interface is represented by a set of integer heights $h_{i}$ residing at the sites $i=1 \ldots L$ of a one-dimensional lattice of length $L$ with periodic boundary conditions, obeying the restriction

$$
h_{i+1}-h_{i}= \pm 1 .
$$

The model is controlled by a single parameter $p \in[0,1]$ and evolves by random sequential updates as follows: Choosing a random site $i$ the height $h_{i}$ is increased by two units with probability $p$ provided that the restriction (8) is not violated. As shown in Fig. 2 this move can be thought of as depositing a diamond. Similarly, the height $h_{i}$ is decreased by two units with probability $1-p$ provided that the restriction (8) is not violated. As usual each local update attempt corresponds to a time increment of $d t=1 / L$. 


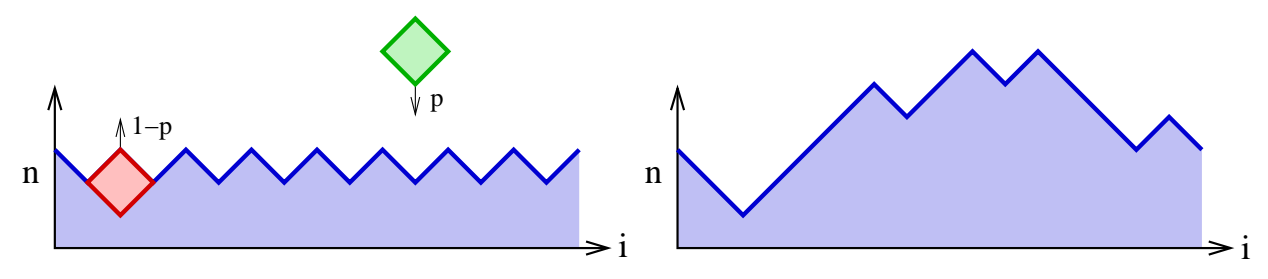

Figure 2. Single step model in $1+1$ dimensions. Left panel: The simulation starts with a flat interface in the form of a horizontal sawtooth pattern. On selecting a site at a local minimum a diamond (rhombus) is deposited with probability $p$, flipping up the interface by two units. Similarly, selecting a site at a local maximum, a diamond evaporates with probability $1-p$, flipping the interface downward by two units. Right panel: Roughening interface after several deposition and evaporation events.

For $p=1 / 2$ the propagation velocity of the interface is zero, detailed balance holds in the stationary state and its dynamics is effectively described by an EdwardsWilkinson equation [23]. For $p \neq 1 / 2$, however, the SSM exhibits KPZ growth with $\lambda$ being proportional to $\frac{1}{2}-p$. In this case the propagation velocity is nonzero and depends on the roughness and the average slope of the interface. For example, the initial velocity of a flat interface (a sawtooth pattern as shown in the left panel of Fig. 2) ) is given by

$$
v(0)=2 p-1
$$

As time proceeds, the interface roughens and the propagation velocity decreases until it saturates at a certain value $v_{L}(\infty)$ which depends on the system size. For the KPZ class the so-called excess velocity $v_{L}(t)-v_{L}(\infty)$ is known to decay with time as $t^{-2 / 3}$ [24]. As a major breakthrough, Prähofer and Spohn recently succeeded in computing the rescaled height profile of a roughening KPZ analytically [25].

In contrast to many other KPZ growth models the SSM allows one to calculate the asymptotic growth velocity $v_{L}(\infty)$ exactly. To this end one identifies segments of positive (negative) slope with particles (vacancies), mapping the single step model onto a partially asymmetric exclusion process (ASEP) [26] of diffusing particles with density $1 / 2$. Since the ASEP is known to evolve towards an uncorrelated product state with a current $j=p / 2-1 / 4$, one can easily show that the propagation velocity of the interface in a finite system of size $L>>1$ tends to

$$
v_{L}(\infty)=\lim _{t \rightarrow \infty} v_{L}(t)=\left(p-\frac{1}{2}\right)\left(1+\frac{1}{L}\right)
$$

In order to study nonequilibrium wetting we need to introduce a hard-core substrate. As there is no parameter in the SSM to control the asymptotic growth velocity of the interface independently from the KPZ nonlinear term $\lambda$, the substrate itself has to move so that we can approach the wetting transition by tuning its velocity $v_{s}$. Obviously, this transition takes place even in finite systems and the critical point is exactly given by $v_{s}=v_{L}(\infty)$. 

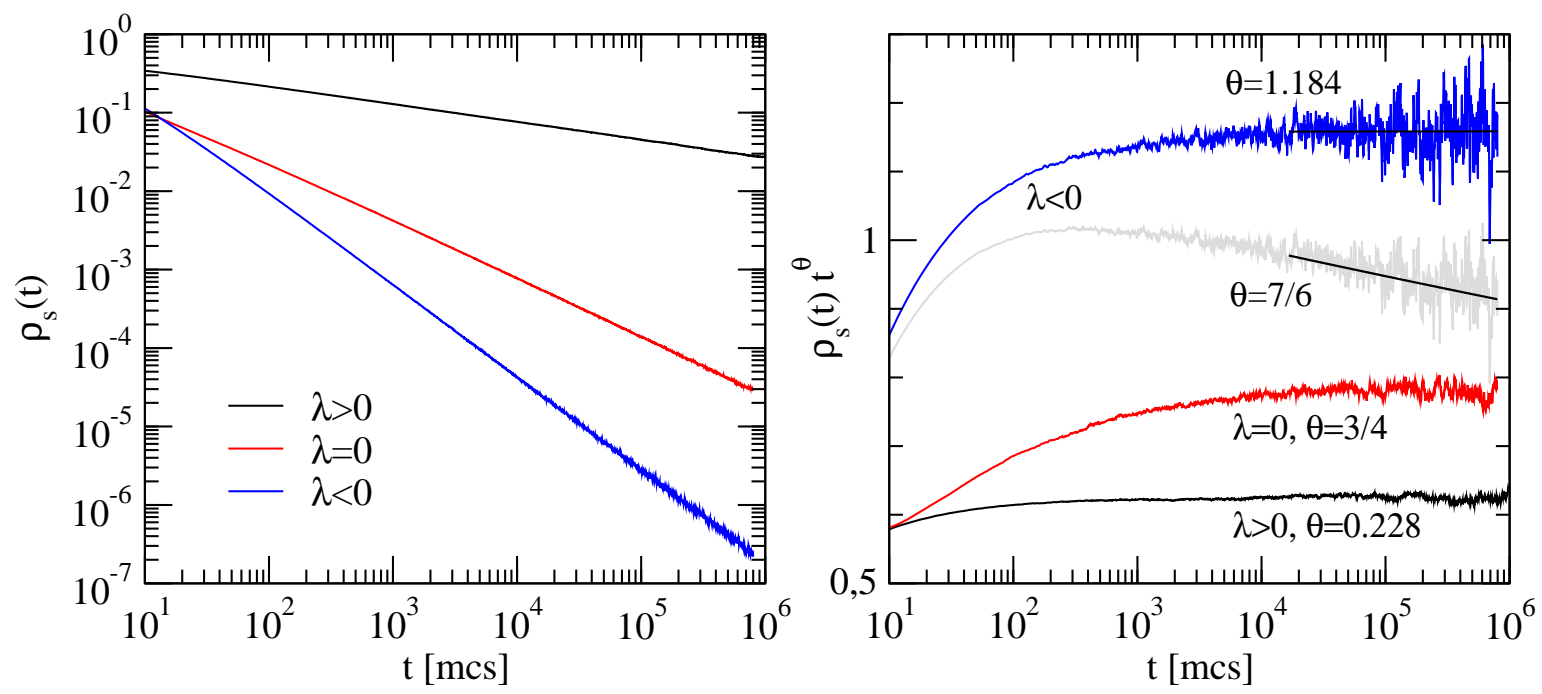

Figure 3. Measurement of the exponent $\theta$ in the single step model. The left panel shows the density of contact points $\rho_{s}(t)$ as a function of time for $p=1(\lambda<0), p=1 / 2$ $(\lambda=0)$, and $p=0(\lambda>0)$. The right panel shows the same data multiplied with the estimated power law $t^{\theta}$. In addition we plotted the curve for $p=1(\lambda<1)$ multiplied by $t^{7 / 6}$, demonstrating that the exponent $\theta=7 / 6$ conjectured by Droz and Lipowski is incompatible with the numerical data.

To determine the surface exponent $\theta$ we simulated the SSM for $p=1 / 2$ as well as for $p=0,1$, where the non-linear effects of the KPZ term are most pronounced. In order to minimize finite-size effects we use a very large lattice size $L=10^{6}$. For $p=1 / 2$ (corresponding to the equilibrium case $\lambda=0$ in the KPZ equation) the interface velocity at the transition vanishes so that the substrate can be implemented by rejecting all updates that would lead to a negative height. The process is then simulated as usual and the density of sites at zero height is averaged over many independent runs. For $p=1$ (corresponding to $\lambda<0$ ) we advance the substrate in temporal intervals $\Delta t=2(1-1 / L)$ by one unit, flipping all sites below the new base line upward. Finally, for $p=0$ (corresponding to $\lambda>0$ ) the substrate moves backward by one unit every interval $\Delta t$, rejecting all updates that would render a height below the actual base line. Since the moving baseline effectively introduces a discrete time step in the algorithm, the density of contact points is measured immediately after (before) moving the wall when $p=1(p=0)$.

The numerical results are shown in Fig. [3. Simulating up to $8 \times 10^{5}$ time steps and averaging over 160 independent runs one obtains convincingly straight lines in a double-logarithmic plot. Extrapolating local slopes we arrive at the estimates

$$
\theta= \begin{cases}0.228(5) & \text { for } p=0 \\ 0.750(5) & \text { for } p=1 / 2 \\ 1.184(10) & \text { for } p=1\end{cases}
$$


In order to illustrate the influence of scaling corrections, we multiplied each data set with $t^{\theta}$ in the right panel of Fig. 3 so that the curves become horizontal for large $t$. Our results have to be compared to the early numerical estimates $\theta_{1}=1.1(1)$ and $\theta_{2}=0.215$ (15) for the MN1 and MN2 Langevin equations [18]. A more recent numerical study of the MN1 equation yielded $\theta_{1}=1.21(3)$ [17].

The numerical result for $p=1$ is particularly interesting as it seems to rule out an earlier conjecture by Droz and Lipowski [20]. Analyzing a synchronization transition between two coupled lattices of tent maps and making use of a hyperscaling relation between stationary and spreading exponents, they proposed that for $\lambda<0$ the exponent $\theta$ should be given by the exact value $7 / 6 \approx 0.1666$. With the accuracy of the present simulations, however, this value lies clearly outside the error margin. To illustrate this difference we plotted $\rho_{s}(t) t^{7 / 6}$ in the right panel of Fig. 3. As can be seen, the curve has a negative slope over at least two decades before finite-size effects set in at $t \approx 10^{5}$. Our result suggests that in both cases of $\lambda \neq 0$ the exponent $\theta$ is probably not a simple rational number, rather it seems to be irrational. For $\lambda=0(p=1 / 2)$, however, our numerical estimate is in fair agreement with $\theta=3 / 4$. In fact, as shown in Appendix A this value can be proven analytically provided that the two-point $C(\ell)$ function of contact points decays algebraically as $\ell^{-\theta z}$.

\section{Gap distribution of an infinite system}

Let us now investigate how the contact points of a critical interface with the substrate are distributed in an infinite system at a given time. Here an important quantity is the gap distribution $G(\ell, t)$ of distances $\ell$ between neighboring contact points measured at time $t$. As Fig. (11) suggests, this distribution depends significantly on the sign of $\lambda$.

Our numerical results are shown in Fig. 4. For $p=0(\lambda>0)$ one observes an ordinary algebraic decay with a cutoff at the correlation length $\xi \sim t^{1 / z}$. This type of decay is the same is in other non-equilibrium critical phenomena as, for example, in DP. For $p \geq 1 / 2(\lambda \leq 0)$, however, the curves first decay algebraically, then reach a plateau, and finally cross over to an exponential cutoff.

The qualitative difference between these two cases is due to the presence of two different length scales, namely, the bulk correlation length $\xi$ and the average gap size $\bar{\ell}$. These two length scales grow with time as

$$
\begin{aligned}
& \xi(t) \sim t^{1 / z} \\
& \bar{\ell}(t) \sim t^{\theta},
\end{aligned}
$$

the latter equation being a consequence of the relation $\bar{\ell}(t)=\frac{1}{\rho_{s}(t)}$ which holds for any point distribution on a one-dimensional line [27]. Depending on the values of the exponents $z$ and $\theta$, two different scenarios are encountered:

- For $\lambda>0(p<1 / 2)$ we have $\theta<1 / z$ and thus the average distance between 

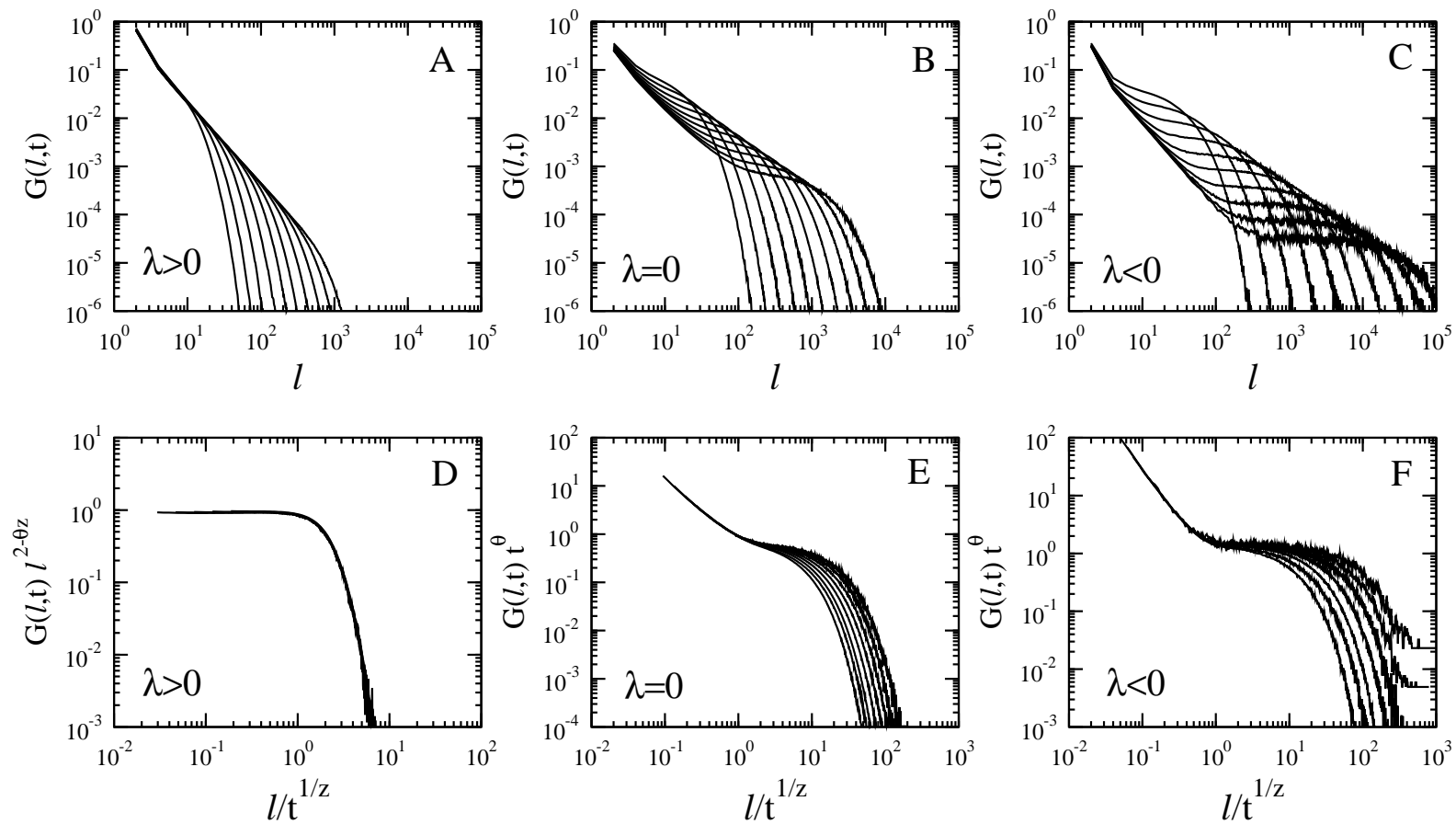

Figure 4. Panels A-C show the gap distribution function depending on the gap size measured in the SSM for $p=0,1 / 2$, and 1 at fixed times $t=16,32,64, \ldots 8192$. Panel D shows a collapse of the data for $\lambda<0$ according to the scaling form (14). Contrarily, panels $\mathrm{E}$ and $\mathrm{F}$ demonstrate that this collapse does not work for $\lambda \leq 0$.

contact points $\bar{\ell}(t)$ is smaller than the correlation length $\xi(t)$. In this case the gap distribution is a simple power law with an exponential cutoff at $\xi(t)$. As in other critical phenomena this suggests the usual scaling form

$$
G(\ell, t) \sim \ell^{-(2-\theta z)} f\left(\frac{\ell}{t^{1 / z}}\right) .
$$

In fact, plotting $G(\ell, t) \ell^{2-\theta z}$ versus $\ell / t^{1 / z}$ one obtains a convincing data collapse, as shown in Fig. 4D.

- For $\lambda \leq 0(p \geq 1 / 2)$, where $\theta>1 / z$, the average distance between contact points is larger than the correlation length. In this case the gap distribution function is found to decay initially as $\ell^{-\theta z}$. Reaching the bulk correlation length $\ell \approx \xi(t)$ it crosses over to an exponential function. However, in contrast to the previous case the associated length scale of this exponential function is $\bar{\ell} \sim t^{\theta}$, hence in a doublelogarithmic graph the curves exhibit a plateau at level $\propto t^{-\theta}$ extending from $\xi(t)$ to $\bar{\ell}(t)$, followed by an exponential cutoff.

In the following Section we will use these postulated scaling forms for the gap distribution to derive the first detachment time in a finite system, showing that the two cases lead to very different types of finite-size scaling. We note that the power-law scaling of the gap distribution is a postulate, supported by numerical results shown in 


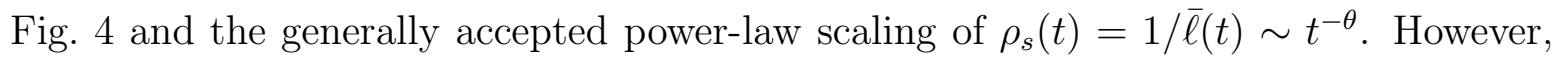
to our knowledge a rigorous proof of power-law scaling in the gap distribution is still missing.

\section{First detachment in a finite system}

We finally turn our attention to finite size scaling analysis, which a common tool to analyze properties of systems at criticality. We recall that the correct order parameter describing the wetting transition is the density $\rho_{s}(t)$ of sites pinned to the substrate, as defined in Eq. (4). Comparison with DP naively leads one to draw an analogy between the pinned sites and the infected sites of systems with an absorbing state (i.e. a state in which the system dynamics get trapped forever). However, in the present case the depinned phase (i.e. the phase in which the substrate is completely covered) is not absorbing in the DP sense. In fact, since the wetting transition takes place at zero relative velocity, fluctuations can easily bring the interface back to the substrate, thus leaving the depinned state. Hence, instead of studying the average survival time $\tau_{a b s}$ (i.e., the average time at which the system becomes trapped in an absorbing state), here we are forced to consider merely the first depinning time $\tau_{f}$, i.e., the average time at which an initially exposed substrate gets completely covered for the first time.

While in systems with an absorbing phase one expects the average survival time in a system of size $L$ to scale as $\tau_{a b s} \sim L^{z}$, where $z$ is the dynamical exponent, our numerical results will show an apparent power law behavior $\tau_{f} \sim L^{\gamma}$, where the exponent $\gamma$ depends on the sign of $\lambda$ and is generally different from the KPZ dynamical exponent. However, a more detailed analysis of the first depinning time probability distribution $P_{L}(t)$ will show that at least for $\lambda \leq 0$ the scaling form

$$
P_{L}(t) \sim L^{-\gamma} g\left(t / L^{z}\right)
$$

is not satisfied, thus indicating that the observed power-law behavior does not hold asymptotically.

\subsection{Numerical results}

In order to measure the first depinning time $\tau_{f}$, we used the single step model with a moving hard-core substrate as described above. To test finite size scaling we simulated various system sizes ranging from $L=64$ to $L=16384$.

For each realization we determined the time $t$ at which the interface was completely depinned for the first time. The resulting histograms of the probability distribution $P_{L}(t)$ of first depinning times are displayed in Fig. 5 . Moreover, we calculated the average depinning time for different system sizes and the relative width of the characteristic 

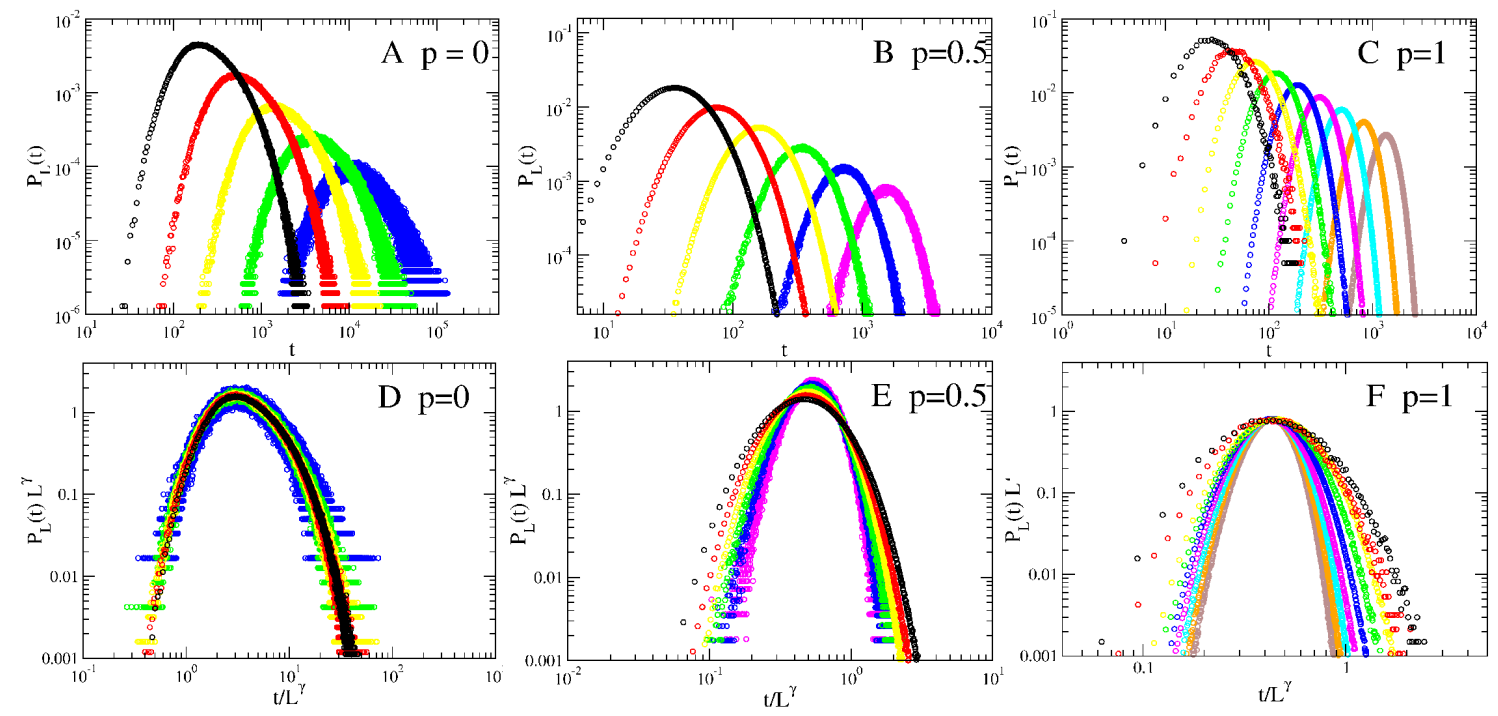

Figure 5. Panels A-C show the distributions of the first depinning times for for $\mathrm{p}=0,0.5$ and 1 . The system size increases from left to right starting with $\mathrm{L}=64$ and going up to $\mathrm{L}=1024,2048$ and 16384 respectively. In panels D-F we test a collapse of the the above distributions according to Eq. (15). Only for $p=0$ the collapse works convincingly.

distributions

$$
\sigma_{\text {rel }}=\frac{\Delta \tau_{f}}{\tau_{f}}
$$

where $\Delta \tau_{f}$ is the standard deviation of first depinning times. The dependence of both quantities on $L$ is shown in Fig. 6. Our numerical results can be summarized as follows:

- In the case of $\lambda>0$ we simulated the SSM with $p=0$ (i.e., no absorption just desorption), averaging over $1.56 \times 10^{6}$ realizations for each system sizes up to $L=1024$. We find that there is a strong indication of an ordinary power law behavior. The average depinning time scales with $L^{\gamma}$, with $\gamma=1.41(2)$, while the probability distributions $P_{L}(t)$ have a constant relative width and, as it is shown in Fig. [6] (panel D), they can be cleanly collapsed according to Eq. (15).

- To study the case $\lambda=0$ and $\lambda<0$ we simulated the SSM with $p=0.5$ and $p=1$ for system sizes ranging from $L=64$ to $L=2048(p=0.5)$ or to $L=16384(p=1)$, averaging over $8 \times 10^{5}$ realizations. In these cases too, the average depinning time $\tau_{f}$ shows an apparent power law scaling. However, the relative width $\sigma_{\text {rel }}$ of the probability distributions shrinks with increasing system size so that the probability distribution curves cannot be convincingly collapsed, making it obvious that we have no ordinary scaling behavior (see Figs. 5 and 6). As it will be shown in the next section, this rather unconventional behavior can be traced back to the particularities of the gap distribution. 

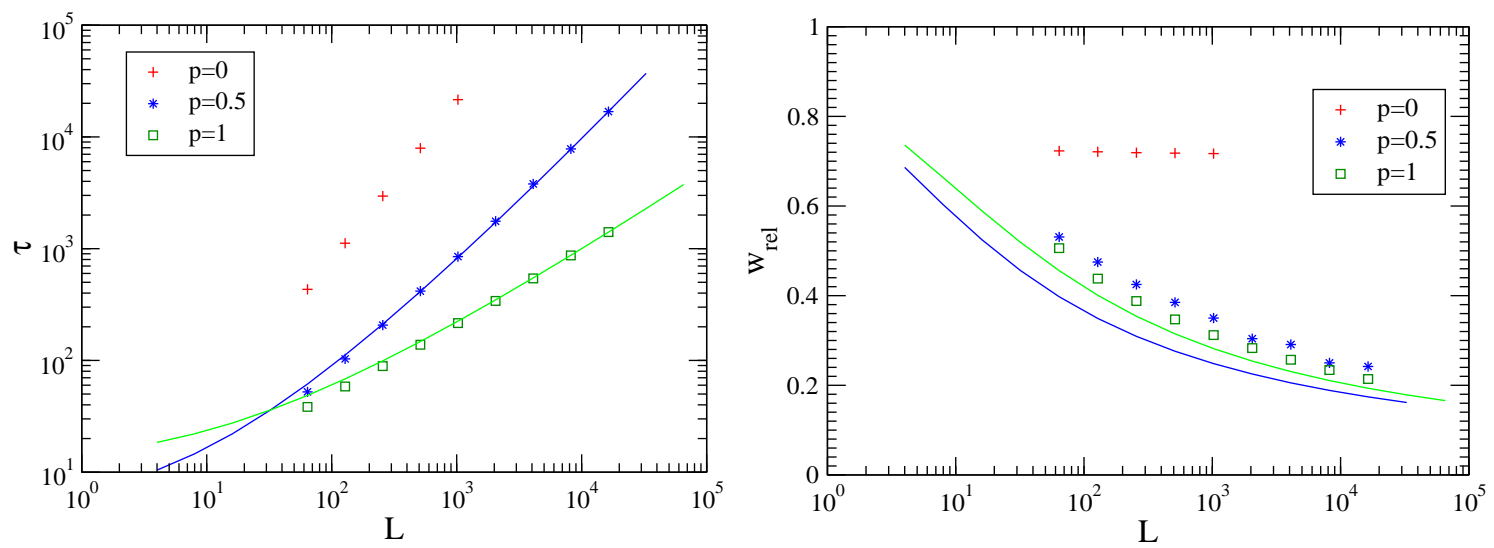

Figure 6. Left: Mean depinning time $\tau_{f}$ plotted against the system size L. Right: Relative width $\sigma_{\text {rel }}$ of the distributions as a function of $L$. As can be seen, the relative width stays constant for $p=0$, in contrast to the cases $p=0.5$ and $p=1$. The solid lines represent the theoretical predictions for the cases $p=0.5$ (blue) and $p=1$ (green) according to Eq. (22).

\subsection{Analytical arguments for the case $\lambda \leq 0$}

In the following we show how the unconventional behavior observed for $\lambda \leq 0$ can be explained analytically in terms of the gap distribution function. The calculation is based on the observation that for $\lambda \leq 0$ the moment of first detachment in a finite system typically takes place long before the correlation length $\xi(t)$ reaches the system size. This means that the interface may be thought of as being divided into $L / \xi(t)$ uncorrelated segments, each of them touching the substrate with a small probability (cf. Fig. (7). The moment of first detachment is then interpreted as the first fluctuation in which all these segments happen to detach independently. This assumption justifies the following approximations:

(a) The distribution of first detachment times in a finite sample of size $L$ with periodic boundary conditions approximately coincides with the corresponding distribution of first detachment for an arbitrarily chosen segment of size $L$ in an infinite system.

(b) The distributions of contact points at different times are assumed to be uncorrelated.

These two assumptions allow us to use the gap distribution investigated in Sect. 3 and to compute the distribution of first detachment times analytically. While assumption (a) is reasonable in systems much larger than the correlation length, assumption (b) is crucial as it neglects all temporal correlations of contact points. 


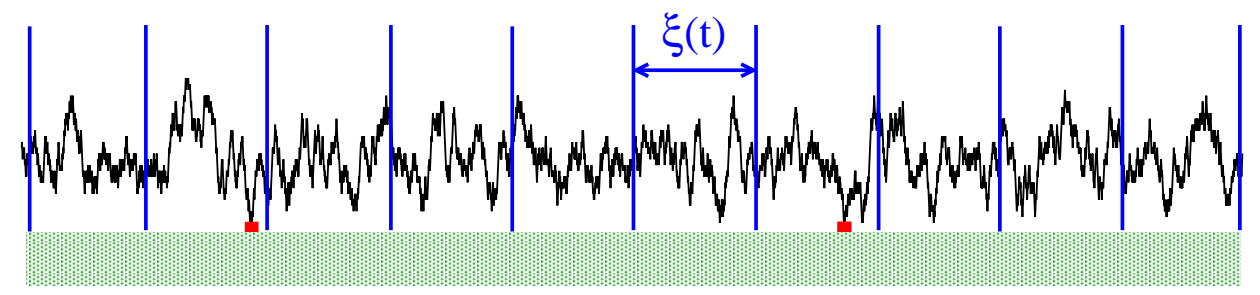

Figure 7. Schematic sketch of a critical interface in a finite system for $\lambda \leq 0$ at a fixed time $t$, divided into mutually uncorrelated segments of size $\xi(t)$. The contact points are marked as red dots. The moment of first detachment corresponds to a fluctuation where all segments independently detach from the surface for the first time.

Obviously, the probability for a segment of size $L$ in an infinite system to detach at time $t$ is given by

$$
Q_{L}^{d}(t)=\frac{\int_{L}^{\infty} d \ell(\ell-L) G(\ell, t)}{\int_{0}^{\infty} d \ell \ell G(\ell, t)},
$$

where $G(\ell, t)$ is the gap distribution discussed in Sect. 3. Consequently, the probability $Q_{L}^{n}(t)$ that the segment never detached up to time $t$ is given by the product of $1-Q_{L}^{d}(t) d t$ over all time steps or - in a continuous formulation - by an exponentiated integral

$$
Q_{L}^{n}(t)=\exp \left(-k \int_{0}^{t} d t^{\prime} Q_{L}^{d}\left(t^{\prime}\right)\right)
$$

where $k$ is a constant to be fitted to the numerical data. The quantity of interest, namely, the probability of first detachment, is then given by

$$
P_{L}(t)=-\frac{d}{d t} Q_{L}^{n}(t)
$$

An examination of these expression reveals that the probability of first detachment is determined by the occasional occurrence of very large gaps. This means that $P_{L}(t)$ is governed by the right edge of the plateau shown in Figs. $4 \mathrm{~B}-4 \mathrm{C}$ while the initial power law decay of the gap distribution has virtually no influence. Therefore, we may approximate the gap distribution function by

$$
G(\ell, t) \simeq A t^{-\theta} \exp \left(-B \frac{\ell}{t^{\theta}}\right)
$$

where $A$ and $B$ are non-universal constants. With this "exponential" approximation the above integrals can be solved, giving

$$
Q_{L}^{d}(t)=\exp \left(-B \frac{L}{t^{\theta}}\right)
$$

and

$$
P_{L}(t)=k \exp \left(-B \frac{L}{t^{\theta}}-\frac{k(B L)^{1 / \theta}}{\theta} \Gamma\left(-1 / \theta, B L / t^{\theta}\right)\right),
$$



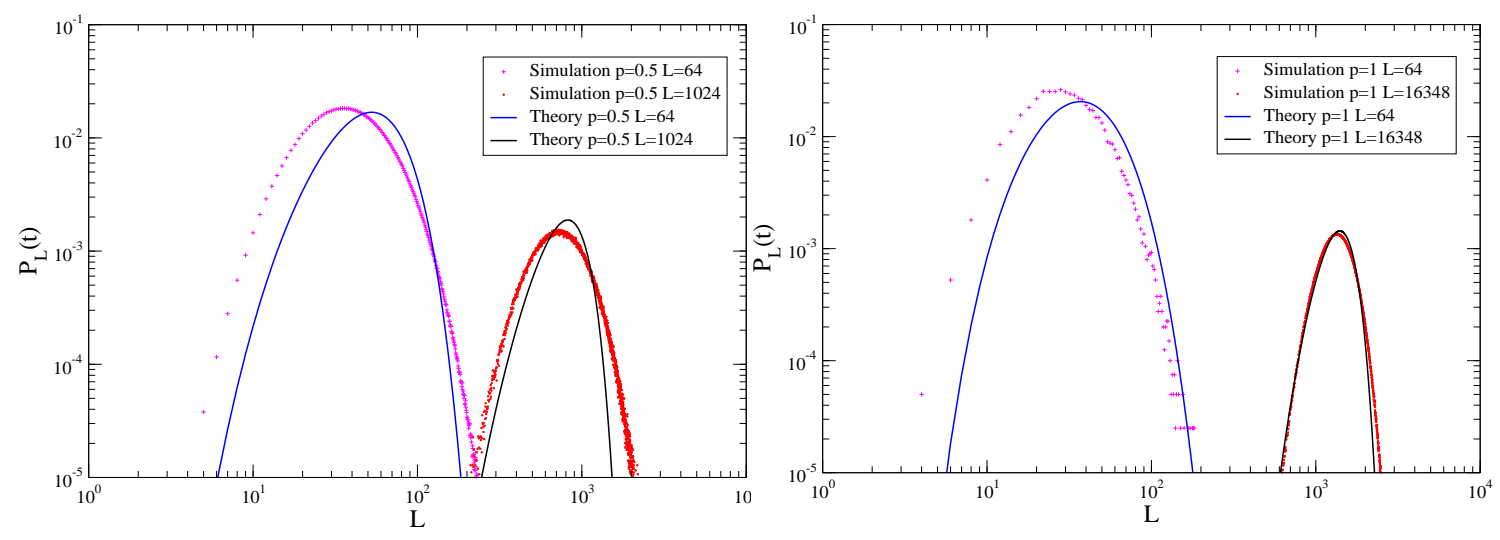

Figure 8. Overlay of analytical predictions for the first depinning time probability distribution for $\lambda \leq 0(p=0.5$ and 1$)$ and numerical results of our simulation on selected system sizes.

where $\Gamma(a, z)=\int_{z}^{\infty} t^{a-1} e^{t} d t$ is the incomplete gamma function. Here the scalinginvariant combination $L / t^{\theta}$ appears in two places whereas the prefactor of the gamma function is proportional to $L^{1 / \theta}$. Obviously it is this prefactor which breaks scaling invariance so that $P_{L}(t)$ does not satisfy the scaling form (15).

In Fig. [6 the average depinning time and the relative width computed numerically from Eq. (22) for $p=1,1 / 2$ are found to be in good agreement with numerical data, while Fig. 8 shows a direct comparison between the analytical and the numerically derived probability distribution functions for the first depinning times. The parameters $B$ and $k$ have been determined by fitting to the numerical data and to grant normalization. Discrepancies between theory and simulations mainly occur for small system sizes and can be traced back to the above approximation of the gap distribution by a exponential function in Eq. (18). As can be seen in Figs. 4B-C the initial powerlaw part of the gap distribution function neglected in the theory is expected to play a more pronounced role for small system sizes. However, for large system sizes, where the "exponential" approximation used to derive Eq. (21) performs better, the agreement steadily improves.

\section{Conclusions}

Nonequilibrium wetting can be modeled by a KPZ growth process combined with a hard-core substrate that gives rise to an additional potential term in the KPZ equation. Adding such a term breaks up-down symmetry and leads to non-trivial surface features, which depend on the sign of the parameter $\lambda$ of the KPZ nonlinear term. For numerical studies of nonequilibrium wetting transitions we advocate the use of the single-step model because of its simplicity and efficency.

With large-scale simulations presented in this paper we have improved numerical estimates of the surface exponent $\theta$. These estimates strongly suggest that $\theta$ takes non- 
trivial irrational values for $\lambda \neq 0$. In particular we can rule out the simple rational value $\theta=7 / 6$ for $\lambda<0$ suggested by Droz and Lipowski. The failure of their argument can probably be traced back to the absence of an absorbing state in the bounded KPZ transition, questioning the feasibility of spreading analysis and hyperscaling relations used in absorbing phase transitions in the present case. However, in the equilibrium case $(\lambda=0)$ our result confirm the simple rational value $\theta=3 / 4$, in agreement with analytical arguments.

In order to investigate finite-size scaling of wetting processes described by the bounded KPZ equation we introduced the average first depinning time as opposed to the average survival time in absorbing phase transitions. In one spatial dimension this naturally leads one to consider the distribution of gaps between pinned sites. We find that two different length scales are shaping the surface dynamics, namely, the spatial correlation length $\xi(t) \sim t^{1 / z}$ and the average gap length $\bar{\ell}(t) \sim t^{\theta}$. If this latter length is larger then $\xi(t)$, as in the case $\lambda \leq 0$, gaps between contact points effectively behave as uncorrelated, thus breaking scale invariance for the first depinning times. This result comes as a surprise if one naively considers the analogy with absorbing phase transitions, where the scaling between temporal and spatial quantities is simply dictated by the dynamical exponent $z$.

The case of $\lambda>0$ on the other hand, shows a clean finite size scaling behavior of the first depinning times. Since in this latter case the spatial correlation length is larger than the average gap size, the analytical arguments presented in Sect. 4.2 no longer apply so that we are not able to predict the shape of the first depinning time probability distribution $P_{L}(t)$. However, we note that our numerical result $\gamma=1.41(2)$ is less then $10 \%$ off the known value for the KPZ dynamical exponent $z=1.5$. Since it has been proven in [18] that spatial and temporal correlations are not asymptotically altered by the additional boundary condition, we expect $\gamma$ to converge gradually versus $z$ in the limit $L \rightarrow \infty$. However, for large but finite systems within the numerically accessible range the estimates may deviate.

\section{Acknowledgments:}

We are grateful to A. Torcini, A. Pikovsky and C. Godrèche for fruitful discussions. Most of the numerical simulations were carried out on the 80-node CIP cluster at the University of Würzburg. We would like to thank A. Klein and A. Vetter for excellent technical support.

\section{Appendix A. Derivation of the exponent $\theta$ in the equilibrium case}

In this Appendix we derive the exponent $\theta$ for the equilibrium case $p=1 / 2$ in the SSM analytically. In this case all transition rates between mutually reachable interface configurations are equal. The system thus approaches a stationary equilibrium state obeying detailed balance in which all interface configurations compatible with the 
restrictions $\left|h_{i}-h_{i+1}\right|=1$ and $h_{i} \geq 0$ occur with the same probability. Although in an infinite system this state is never reached, a well-defined quantity is the conditional correlation function

$$
c(\ell)=\frac{\left\langle\delta_{h_{i}, 0} \delta_{h_{i+\ell}, 0}\right\rangle}{\left\langle\delta_{h_{i}, 0}\right\rangle}
$$

where $\langle\cdot\rangle$ denotes the spatial average over the index $i$. This correlation function may be interpreted as the conditional probability to find site $i+\ell$ at zero height given that site $i$ is a contact point, too. As the interface roughens, numerator and denominator tend to zero but their quotient tends to a well-defined non-zero value.

The present proof relies on the assumption that this two-point function - like other two-point functions in scale-free situations - decays according to the power law

$$
c(\ell) \sim \ell^{-\theta z}
$$

where $z=2$ is the dynamical exponent. As will be shown, the exponent $\theta$ can be computed by deriving an exact expression for $c(\ell)$.

Adopting a transfer matrix approach used in Ref. [28, 29], the correlation function A.1 may be expressed as

$$
C(\ell)=\frac{\left\langle 1\left|T^{\ell}\right| 1\right\rangle}{\Lambda^{\ell}}
$$

where $T$ is an infinite-dimensional transfer matrix with matrix elements

$$
T_{h, h^{\prime}}=\left\{\begin{array}{ll}
1 & \text { if }\left|h-h^{\prime}\right|=1 \\
0 & \text { otherwise }
\end{array} \quad h, h^{\prime} \in\{0,1,2, \ldots\}\right.
$$

while $\Lambda=-2$ its dominating eigenvalue and $\langle 1|=| 1\rangle^{\dagger}=(1,0,0, \ldots)$ denotes the vector representing height zero. Because of the special structure of $T$, where only the secondary diagonals are occupied, the evaluation of Eq. (A.3) reduces to counting all possible paths of the interface from site $i$ to site $i+\ell$. The number of such paths is given by Catalan numbers, leading to the exact solution

$$
C(\ell)= \begin{cases}\frac{\ell !}{(\ell / 2) !(\ell / 2+1) ! 2^{\ell}} & \text { if } \ell \text { even } \\ 0 & \text { if } \ell \text { odd }\end{cases}
$$

Inserting Stirling's formula $n ! \simeq \sqrt{2 \pi} n^{n+1 / 2} e^{-n}$ it straight forward to show that this correlation function decays asymptotically as

$$
c(\ell) \simeq \sqrt{\frac{8}{\pi}} e \ell^{-3 / 2}
$$

Together with the known exponent $z=2$ we thus arrive at $\theta=3 / 4$. 


\section{References}

[1] S. Dietrich, in "Phase Transitions and Critical Phenomena", (C. Domb and J. L. Lebowitz, eds.), Vol. 12, p. 1. Academic Press, London and Orlando.

[2] D. S. Fisher and D. A. Huse, Phys. Rev. B 32, 247 (1985); D. M. Kroll and R. Lipowsky, Phys. Rev. B 26, 5289 (1982); E. Brézin, B. I. Halperin, and A. Leibler, Phys. Rev. Lett. 50, 1387 (1983).

[3] Y. Tu, G. Grinstein and M. A. Muñoz Phys. Rev. Lett. 78, 274 (1997); M. A. Muñoz and T. Hwa, Europhys. Lett. 41, 147 (1998).

[4] H. Hinrichsen, R. Livi, D. Mukamel, and A. Politi, Phys. Rev. Lett. 79, 2710 (1997).

[5] H. Hinrichsen, R. Livi, D. Mukamel, and A. Politi, Phys. Rev. E 61, R1032 (2000).

[6] L. Giada and M. Marsili, Phys. Rev. E 62, 6015 (2000).

[7] J. Candia and E. V. Albano, Eur. J. Phys. B 16, 531 (2000).

[8] F. de los Santos, M. M. T. da Gama, and M. A. Muñoz, Europhys. Lett. 57, 803 (2002); F. de los Santos, M. M. T. da Gama, and M. A. Muñoz, Phys. Rev. E 67, 021607 (2003).

[9] H. Hinrichsen, R. Livi, D. Mukamel, and A. Politi, Phys. Rev. E 68, 041606 (2003).

[10] M. Kardar, G. Parisi and Y-C Zhang, Phys. Rev. Lett. 56889 (1986).

[11] A.-L. Barabási and H. E. Stanley, Fractal Concepts in Surface Growth, Cambridge University Press, Cambridge (1995).

[12] L. Baroni, R. Livi, and A. Torcini, Phys. Rev. E 63, 036226 (2001).

[13] V. Ahlers and A. Pikovsky, Phys. Rev. Lett. 88254101 (2002).

[14] A. Pikovsky and A. Politi, Nonlinearity 11, 1049 (1998).

[15] Y. Tu, G. Grinstein and M.A Munõz, Phys. Rev Lett. 78, 274 (1997).

[16] M. A. Muñoz, F. de los Santos, and A. Achahbar, Braz. J. Phys. 33, 443 (2003).

[17] Ivan Dornic, Hugues Chate, and M.A. Muñoz, eprint cond-mat/0404105.

[18] M. A. Muñoz, Nonequilibrium Phase Transitions and Multiplicative Noise, in "Advances in Condensed Matter and Statistical Mechanics", ed. by E. Korutcheva and R. Cuerno, Nova Science Publishers, 2004.

[19] F. Ginelli and H. Hinrichsen, J. Phys A 37, 11085 (2004).

[20] M. Droz and A. Lipowski, Phys. Rev. E 67, 056204 (2003).

[21] F. Ginelli, V. Ahlers, R. Livi, D. Mukamel, A. Pikovsky, A. Politi, and A. Torcini, Phys. Rev. E 68, 065102(R) (2003).

[22] H. Hinrichsen, Adv. Phys. 49, 815 (2000) cond-mat/0001070.

[23] S.F. Edwards and D.R. Wilkinson, Proc. R. Soc. London A 381, 17 (1982).

[24] J. Krug and P. Meakin, J. Phys. A: Math. Gen. 23, L987 (1990)

[25] M. Prähofer and H. Spohn, Phys. Rev. Lett. 84, 4882-4885 (2000); Physica A 279, 342 (2000); J. Stat. Phys. 1081071 (2002); J. Stat. Phys. 115255 (2004).

[26] T. M. Ligget, Interacting Particle Systems, Springer-Verlag, New York 1985.

[27] See for instance the brief discussion in: F. Ginelli, H. Hinrichsen, R. Livi, D. Mukamel, and A. Politi, Phys. Rev. E 71026121 (2005).

[28] J. M. J. van Leeuwen and H. J. Hilhorst, Physica A 107, 318 (1981).

[29] T. W. Burkhardt, J. Phys. A 14, L63 (1981). 